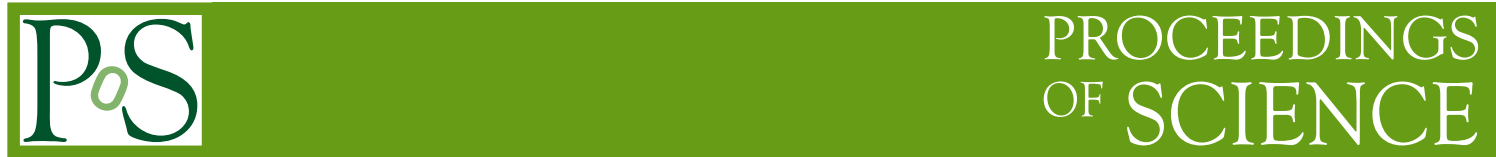

\title{
Z+Jets results from CDF
}

\author{
Costas VELLIDIS **i \\ FNAL \\ E-mail: vellidis@fnal.gov
}

\section{Stefano Camarda}

Universitat Autonoma de Barcelona

E-mail: camarda@ifae.es

\section{Mario Martinez-Perez}

Universitat Autonoma de Barcelona

E-mail: mmp@ifae.es

\section{Lorenzo Ortolan}

Universitat Autonoma de Barcelona

E-mail: loreorto@ifae.es

\section{Veronica Sorin}

Universitat Autonoma de Barcelona

E-mail: vsorineifae.es

We present results of cross section measurements for $\mathrm{Z} / \gamma^{*}$ production, where the $\mathrm{Z} / \gamma^{*}$ boson decays leptonically, associated with light-flavor jets and with a bottom-flavor jet. The measurements use the full CDF Run II data sample. The results are compared with state-of-the-art calculations. The comparisons show generally good agreement between data and theory.

36th International Conference on High Energy Physics

4-11 July 2012

Melbourne, Australia

\footnotetext{
${ }^{*}$ Speaker.

On behalf of the CDF Collaboration.
} 


\section{Introduction}

The reported measurements are a Tevatron legacy, testing perturbative quantum chromodynamics (pQCD), the theory of strong interaction, at high momentum transfer squared $\left(Q^{2}\right)$ and aiming to reduce the large theoretical uncertainty in the $\mathrm{Z} / \gamma^{*}+$ heavy-flavor jet production. They constrain predictions of $\mathrm{Z} / \gamma^{*}+$ jets production rates and shapes, which is important because $\mathrm{Z} / \gamma^{*}+$ jets production is a sizable background in studies of rare standard model processes, such as top-quark, diboson, and Higgs boson production, and in searches for new physics.

\section{Data analysis}

The $\mathrm{Z}$ boson (or time-like virtual photon) is reconstructed from $l^{+} l^{-}$pairs $(l=e$ or $\mu)$ with transverse momentum $p_{T}>25 \mathrm{GeV} / c$ with respect to the beam axis, rapidity $|y|<1.0$, and invariant mass $66<M_{l l}<116 \mathrm{GeV} / c^{2}$. Jets are reconstructed using the midpoint algorithm [1] in a cone of radius $R=0.7$ in the $\eta-\phi$ space and are required to have $p_{T}>30 \mathrm{GeV} / c,|y|<2.1$, and $\Delta R(l$, jet $)>0.7$. Differential cross sections are measured independently in the electron and muon channels and then combined accounting for correlations between systematic uncertainties with a modified best linear unbiased estimator (BLUE) algorithm [2] that implements asymmetric errors. The measured cross sections are corrected to hadron level. The total systematic uncertainty ranges from $5 \%$ to $15 \%$, with the jet energy scale being the dominant source of uncertainty. For the $\mathrm{Z} / \gamma^{*}+$ heavy flavor measurements, a secondary vertex algorithm is used to tightly tag the heavyflavor jets, the second electron in $\mathrm{Z} / \gamma^{*} \rightarrow e^{+} e^{-}$decays can be either central $(|y|<1.0)$ or forward $(1.2<|y|<2.8)$ to increase the acceptance, and an artificial neural network (ANN) is implemented to increase the electron and muon identification efficiency. The bottom flavor fraction is determined by fitting simulated templates to the measured invariant mass distribution of the tagged jets and the measured $\mathrm{Z} / \gamma^{*}+$ heavy flavor cross section is normalized to the inclusive $\mathrm{Z} / \gamma^{*}$ cross section or the $\mathrm{Z} / \gamma^{*}+$ light flavor cross section in order to reduce systematic uncertanties.

\section{3. $\mathrm{Z} / \gamma^{*}+$ light-flavor jets production}

The results are compared with predictions from the "tree-level" matrix-element program ALPGEN [3], which calculates the $\mathrm{Z} / \gamma^{*}+$ jets production amplitudes for any given jet multiplicity without accounting for virtual-particle "loop" processes, and from the program POWHEG [4], which calculates the $\mathrm{Z} / \gamma^{*}+$ jets production amplitudes at next-to-leading order (NLO) in the coupling parameter of the strong interaction. In both cases, the partons (quarks and gluons) generated by the matrix-element program are showered using the standard parton-showering program PYTHIA [5], which also implements a realistic "underlying event" from soft parton interactions around the hard scattering process, matching $\mathrm{pQCD}$ and non-pQCD physics. Comparisons with more calculations are available in the public Web site of the analysis [6]. The largest theoretical uncertainty originates from the choice of the scale. Uncertainties from the parton distribution functions (PDF), of the order of $2-4 \%$, are also considered.

Figures 1 and 2 show the comparisons of the measured and predicted cross sections as functions of the transverse momentum of the reconstructed boson and of the jet multiplicity, respectively. Good agreement between data and predictions is observed, both in the normalizations and 
in the shapes of the distributions. The ALPGEN + PYTHIA calculation is more sensitive to the choice of the PYTHIA tune and has larger scale and matching uncertainties.

\section{4. $\mathrm{Z} / \gamma^{*}+$ bottom-flavor jet production}

The results are compared with predictions from the NLO matrix-element program MCFM [7]. Comparisons with more calculations, including ALPGEN + PYTHIA, are available in the public Web site of the analysis [8]. The fraction of the cross section, integrated over the acceptance, for $\mathrm{Z} / \gamma^{*}+$ bottom flavor production to the cross section for inclusive $\mathrm{Z} / \gamma^{*}$ production is $\left(0.261 \pm 0.023_{\text {stat }} \pm 0.029_{\text {syst }}\right) \%$ and to the cross section for $\mathrm{Z} / \gamma^{*}+$ light flavor production is $(2.08 \pm$ $\left.0.18_{\text {stat }} \pm 0.27_{\text {syst }}\right) \%$. The corresponding fractions from MCFM are, respectively, $0.23 \%$ and $1.8 \%$ at $Q^{2}=m_{Z}^{2}+\left\langle p_{T, Z}\right\rangle^{2}$, and $0.29 \%$ and $2.2 \%$ at $Q^{2}=\left\langle p_{T, j e t}\right\rangle^{2}$, where $m_{Z}$ and $\left\langle p_{T, Z}\right\rangle$ are the mass and the average transverse momentum of the $\mathrm{Z}$ boson, respectively, and $\left\langle p_{T, j e t}\right\rangle$ is the average transverse momentum of the bottom-flavor jet. The comparison shows that both fractions are well predicted by MCFM within uncertainties. Figure 3 shows the comparisons of the measured and predicted cross sections as functions of the transverse momentum and the rapidity of the bottom-flavor jet. Reasonable agreement between the data and the predictions is observed within uncertainties.

\section{Summary}

We report high precision measurements of the cross sections for $\mathrm{Z} / \gamma^{*}$ production, where the $\mathrm{Z} / \gamma^{*}$ boson decays leptonically, associated with light-flavor jets and with a bottom-flavor jet. Both measurements use the full CDF data sample. The results are compared with state-of-the-art calculations. The comparisons show good agreement between the data and NLO pQCD predictions. Calculations at the "tree level" also agree with the data, but have larger scale and matching uncertainties and stronger dependence on the non-pQCD physics tune.

\section{References}

[1] S. D. Ellis, J. Huston, and M. Tönnesmann, eConf C010630, PS13 (2001); arXiv:hep-ph/0111434.

[2] A. Valassi, Nucl. Instrum. Methods A 500, 391 (2003).

[3] M. L. Mangano, M. Moretti, F. Piccinini, R. Pittau, and A. D. Polosa, J. High Energy Phys. 0307, 001 (2003).

[4] S. Alioli, P. Nason, C. Oleari, and E. Re, J. High Energy Phys. 07, 060 (2008).

[5] T. Sjöstrand, P. Eden, C. Friberg, L. Lombard, G. Miu, S. Mrenna, and E. Norrbin, Comp. Phys. Comm. 135, 238 (2001).

[6] http://www-cdf.fnal.gov/physics/new/qcd/zjets10fb_new/

[7] J. M. Campbell and R. K. Ellis, Phys. Rev. D 60, 113006 (1999).

[8] http://www-cdf.fnal.gov/physics/new/qcd/zbjet2012/ 


\section{CDF Run II Preliminary}
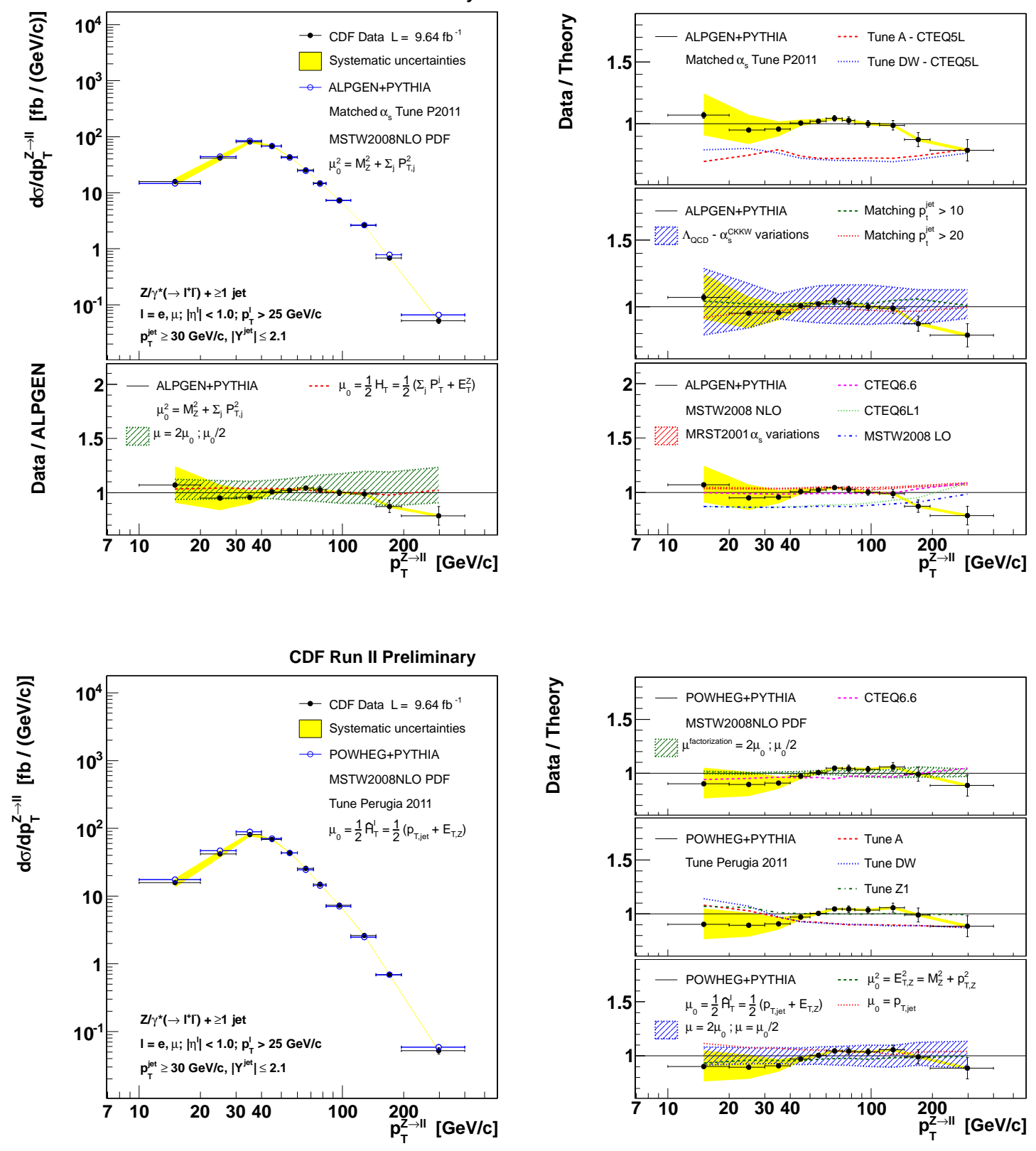

Figure 1: Differential cross sections (left) and data/theory ratios (right) for $\mathrm{Z} / \gamma^{*}+$ light flavor production as functions of the transverse momentum of the $\mathrm{Z}$ boson. The data are compared with predictions from the "tree-level" ALPGEN + PYTHIA (top) and NLO POWHEG + PYTHIA (bottom) calculations. 

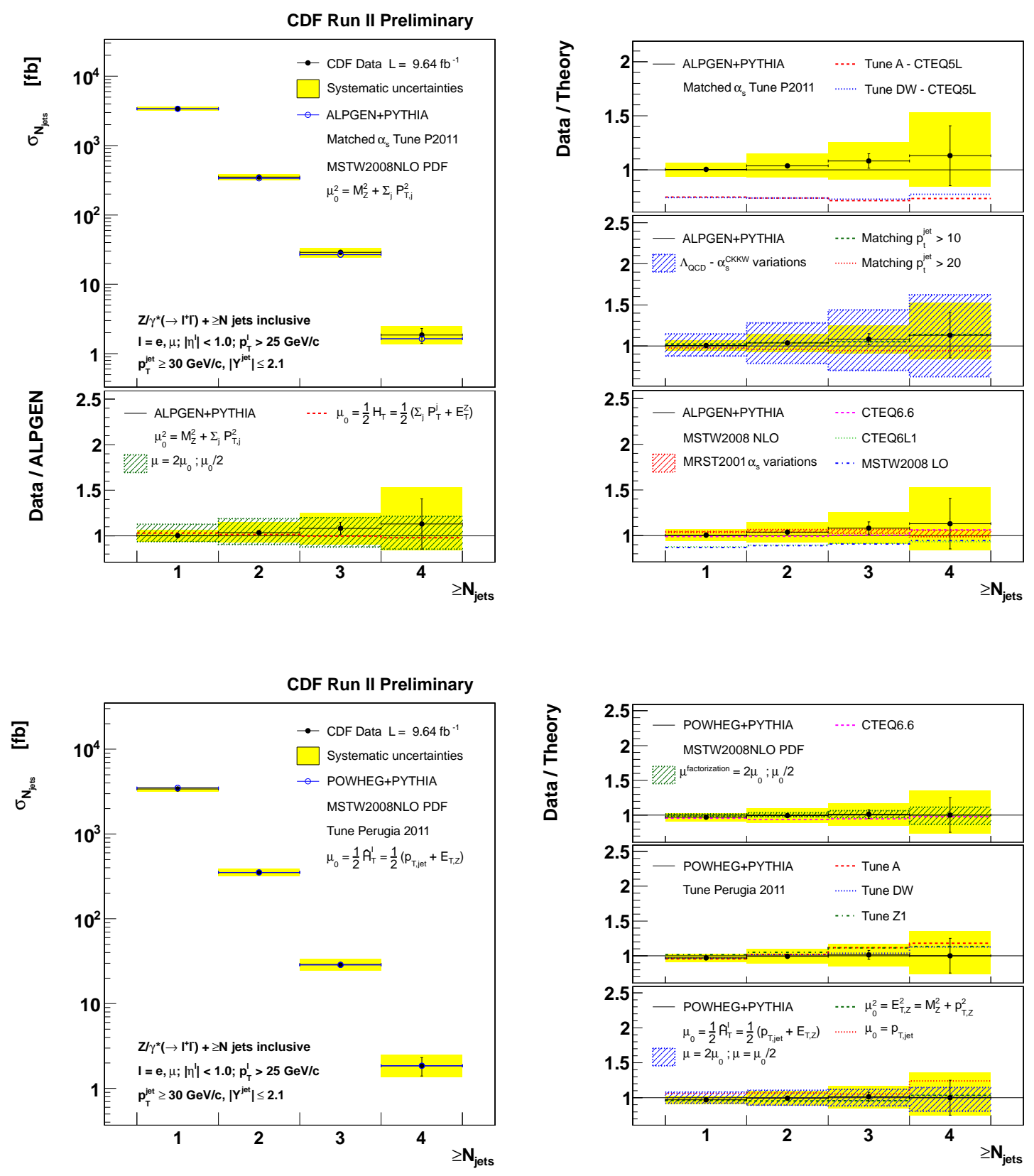

Figure 2: Differential cross sections (left) and data/theory ratios (right) for $\mathrm{Z} / \gamma^{*}+$ light flavor production as functions of the jet multiplicity $\geq N_{\text {jets }}$, which means at least $N_{\text {jets }}$ jets in the event. The data are compared with predictions from the "tree-level" ALPGEN + PYTHIA (top) and NLO POWHEG + PYTHIA (bottom) calculations. 

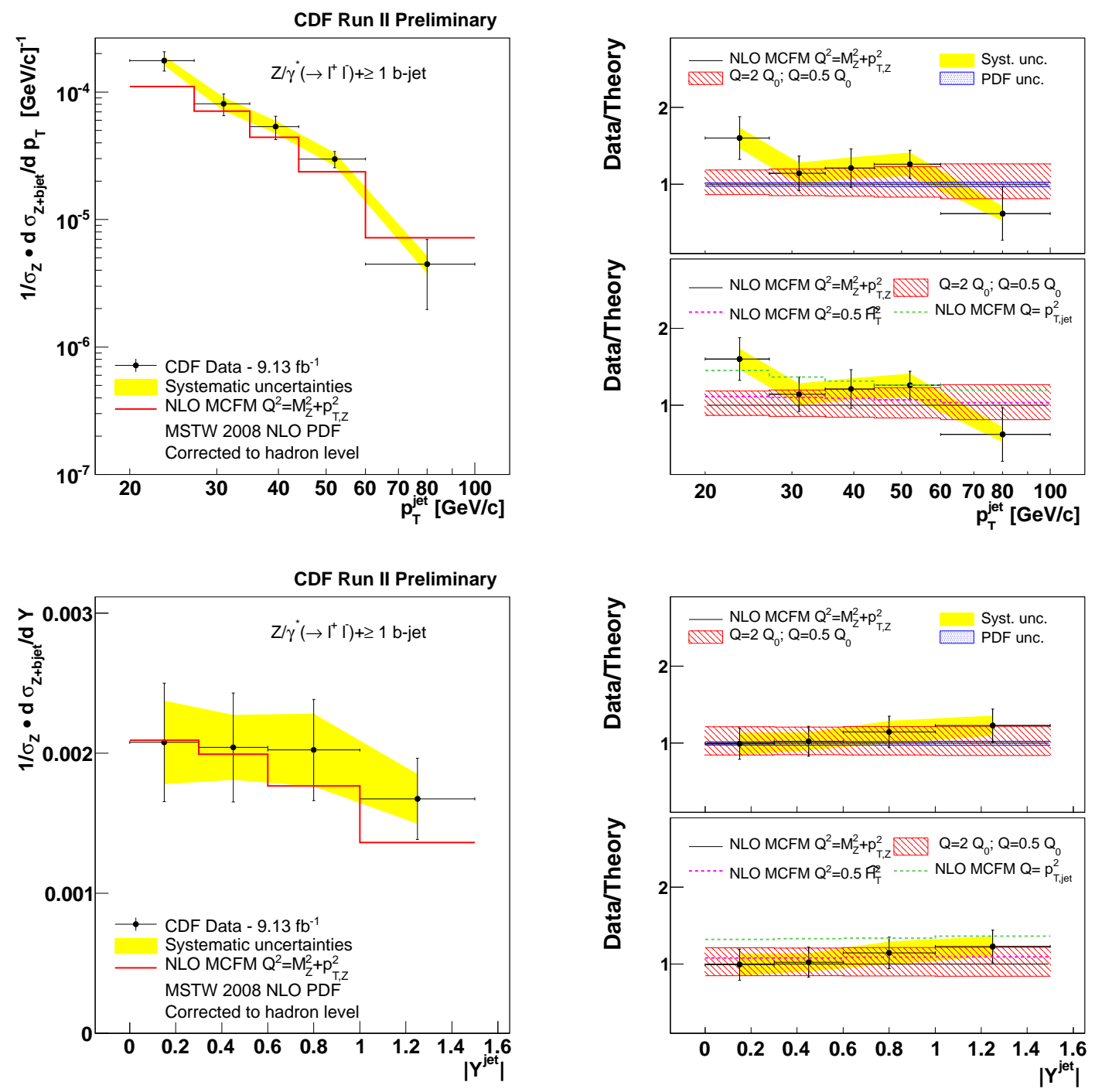

Figure 3: Differential cross sections (left) and data/theory ratios (right) for $\mathrm{Z} / \gamma^{*}+$ bottom flavor production as functions of the b-jet transverse momentum (top) and rapidity (bottom). The cross sections are normalized to the cross section for inclusive $\mathrm{Z} / \gamma^{*}$ production integrated over the acceptance. The data are compared with NLO MCFM calculations for various choices of the scale. 